\title{
El desarrollo comunitario en la obra de los teóricos más representativos
}

\author{
ANDRÉS DEL MORAL VICO \\ Universidad de Granada
}

\begin{abstract}
SUMMARY.- Some time has passed since the sistematization and the theory on the Community Development process were begun. Meanwhile, concepts, terms which denote it, points of view, etc. have been modified.

At present they have appeared a series of expressions related with as many socieducative interventions which often share common action spaces. This situation becomes more complicate because each one of the interventions uses specific methodologies which come to be added to the wide conceptual spectrum now existent.

This reality requires a contextual analysis in order to clarify somewhat the panorama and to obtain and agreement on these which could be considered as Community Development and every term close to it.

Between others, a strategy is to study the works of most representatives theorist in this subject. We have divided the different theoretical contributions as it follows:

a) Latinoamerican theorists.

b) European theorists.

c) Spanish theorists.
\end{abstract}

\section{APORTACIONES DE LOS TEÓRICOS}

Una vía de aproximación al ámbito conceptual del Desarrollo Comunitario, bajo nuestro punto de vista, consistirá en examinar la obra escrita de los principales autores que han realizado investigaciones en esta materia.

Esta tarea no es fácil. Son muchos los estudiosos que se han centrado en la investigación teórica y práctica de estos procesos. Teresa Porzecanski $(1983,37)$ nos confirma esta realidad. De la misma manera, César Rodríguez (1970) (cf. Porcekanski, 1983, 38-39) nos ratifica la atención que este campo del conocimiento ha despertado a lo largo de los años.

Nosotros nos centraremos en los autores más próximos en el espacio y en el tiempo, fundamentalmente en aquellos que nos han servido de soporte teórico en nuestro trabajo. Por otro lado, pretendemos no extendernos excesivamente en este apartado, sólo lo suficiente para alcanzar el objetivo de estas aportaciones: la clarificación conceptual del objeto de nuestra investigación. 


\section{Teóricos del área latinoamericana}

Justo es que comencemos esta ronda de teóricos con Ezequiel Ander-Egg, prolífero autor en esta temática. Su obra escrita ha sido tenida muy en cuenta en este estudio.

En el prefacio de una de sus obras más conocidas (1986a, 10) nos avanza una primera definición de desarrollo de la comunidad: parte integrante del concepto más amplio, más general y más complejo del «Desarrollo», y lo entendemos como método y técnica que contribuye positiva, real y efectivamente en un proceso de Desarrollo integral y armónico, atendiendo fundamentalmente a ciertos aspectos extra-económicos, en particular psico-sociales, que intervienen en la promoción de actitudes, aspiraciones y deseos para el desarrollo.

Más adelante (1986a, 69-72), sintetiza las notas distintivas del desarrollo de la comunidad:

a) Se trata de una técnica o práctica social.

b) Difiere de otras técnicas por sus objetivos, modalidad operativa y nivel en que funciona.

c) Las actitudes que genera en la población son más importantes que el contenido material de los proyectos.

d) La participación popular se convierte en principio axial.

e) Existe un sustrato ideológico-político como componente teleológico orientador de esta técnica.

No obstante, una de las aportaciones más valiosas de Ander-Egg es su labor recopiladora centrada en teorías y prácticas de desarrollo comunitario que creemos interesante traer aquí.

Advierte el investigador argentino que las actividades de ayuda mutua, de acción conjunta, son tan antiguas como la misma humanidad (1986a, 47). Tanto en las sociedades no industrializadas como en la mayoría de los pueblos subdesarrollados, se producen una serie de hechos que giran alrededor de la idea de colaboración, participación, ayuda, germen de lo que más tarde se va a llamar desarrollo de la comunidad.

Basándose en la documentación más sobresaliente de diferentes organizaciones internacionales, este autor nos ofrece en su obra un estudio evolutivo sobre la conceptualización que nos ocupa. Al mismo tiempo, en este trabajo recoge una síntesis de las opiniones más consagradas de otros teóricos respecto a los conceptos de organización de la comunidad y desarrollo de la comunidad.

Sobre la expresión «ORGANIZACION DE LA COMUNIDAD», que se empleó con anterioridad a la de desarrollo, recoge las siguientes aportaciones (AnderEgg, 1986a, 61):

En 1921, E. C. LINDEMAN: esfuerzo consciente de parte de la comunidad para controlar sus problemas y lograr mejores servicios de especialistas, organizaciones e instituciones.

En 1925, W. PETTIT: la forma de ayudar a un grupo de personas a reconocer sus necesidades comunes y a resolverlas. 
En 1930, J. F. STEINER: modo organizado de trabajar para resolver conflictos y hacer posible un progreso consistente.

En 1939, D. SANDERSON y R. POLSEN: desarrollar relaciones entre grupos e individuos que permita a éstos actuar conjuntamente a los fines de crear y sostener facilidades y agencias mediante los cuales alcanzar sus más altos valores para el bienestar general de la comunidad.

En 1940, A. JoHNSON: el arte y el proceso de desarrollar los recursos potenciales y el talento de los individuos que componen la comunidad.

En 1942, L. W. MAYO: proceso por el cual se trata de establecer un equilibrio entre las necesidades y los recursos.

En 1943, A. DunHAM: proceso dirigido a la creación de recursos habida cuenta de las necesidades dentro de una determinada área geográfica o de un campo específico del servicio social.

A partir de 1948 se comienza a utilizar la expresión desarrollo de la comunidad para denominar estos procesos. Ander-Egg (1986a, 63), recopila en su obra las aportaciones más interesantes:

La Colonial Office Británica lo entiende como un programa gubernamental para el mejoramiento global de la vida de una comunidad con su participación activa (año 1948).

La $O E A$ : técnica o proceso que emplea el servicio social para suscitar la racional participación de los integrantes de una determinada zona o población, en una empresa de mejoramiento individual y de progreso cuiectivo, sobre la base de los propios recursos (año 1950).

Naciones Unidas: proceso mediante el cual los miembros de una comunidad discuten cuidadosamente, primero definen lo que quieren y después planean y actúan en conjunto para satisfacer sus deseos (año 1953).

Caroline Ware (1954): un proceso para suscitar grupos funcionales de ciudadanos capaces de ser los agentes activos y responsables de su progreso, usando para ello como medios: la investigación en común de los problemas locales, el planeamiento y la ejecución por sí mismos de las soluciones que antes convinieron, y la coordinación voluntaria con los demás grupos y con las autoridades oficiales, de modo que se obtenga el bienestar total de la comunidad.

Pasemos a otro autor del área latinoamericana. Se trata en esta ocasión de una mujer, Teresa Porzecanski, que a su labor docente en la universidad de la República Oriental del Uruguay y otras universidades extranjeras hay que sumar su larga experiencia como trabajadora social en programas de desarrollo comunitario en varios países.

En el capítulo primero de una de sus obras más difundidas (Porzecanski, 1983, 32) da una definición de desarrollo de la comunidad para indicar el alto grado de abstracción de sus objetivos: conjunto de acciones destinadas a provocar un cambio orientado de conductas a nivel de un microsistema social, participativo y que signifique una etapa más avanzada de progreso humano. Hace unas interesantes observaciones aprovechando la elevada generalidad de las aspiraciones con objeto de poner en su justo término lo que verdaderamente puede y no puede alcanzar el desarrollo comunitario. 
Es en el capítulo segundo donde encontramos las apreciaciones que mejor pueden ubicarse en este apartado de nuestro trabajo, sobre todo en el epígrafe que titula a la búsqueda de los conceptos (Porzecanski, 1983, 37).

Como ya indicábamos más arriba, comienza el capítulo aludiendo a la gran cantidad de estudios realizados sobre Organización y Desarrollo de la Comunidad. Entre las muchas definiciones generadas, la autora hace una selección que creemos conveniente recoger por razones más que repetidas.

«Organización de la Comunidad es un esfuerzo deliberado dirigido a ayudar a los grupos a alcanzar unidad de propósito y acción hacia objetivos generales y específicos» (MacMillen, 1947).

«Organización de la Comunidad es el proceso mediante el cual una comunidad identifica sus necesidades y objetivos, los ordena o jerarquiza, desarrolla la confianza y el deseo de hacer algo ante ellos, procura los recursos (internos o externos) para tratarlos, emprende la acción al respecto y desarrolla las actitudes y prácticas de cooperación y colaboración dentro de la comunidad» (Murray Ross, 1955).

También recoge las definiciones del Seminario de la OEA del año 1951 y algunas más de Naciones Unidas, todas ya consideradas por nosotros y que no vamos a repetir.

La autora entresaca cuatro elementos básicos de estas definiciones (Porzecans$\mathrm{ki}$, 1983, 38):

a) Se trata de un trabajo participativo de grupos comunitarios.

b) Su finalidad sería un mejoramiento colectivo y de bienestar.

c) Esa finalidad se cumpliría por los propios interesados.

d) Se integraría la acción de éstos a la acción del gobierno local y nacional.

En el mismo capítulo, cita la investigación de César Rodríguez (1970) quien después de analizar veinticinco definiciones sobre Organización y Desarrollo de la Comunidad concluye: el $76 \%$ de todas ellas habla de fuerzas horizontales y verticales en el sentido de ayuda estatal. El $68 \%$ alude a la participación de la población. El 64\% comparten el significado común de auto-ayuda y asistencia mutua por parte de los interesados en relación a la solución de sus propios problemas. El $80 \%$ encuentran importante el mejoramiento de las condiciones de vida, tanto materiales como espirituales.

Basándose en los estudios de otros autores (Grazziosi, Scaron de Quintero, etc.), señala un dato interesante, el nivel de relatividad existente en los términos más destacados de las definiciones de los teóricos. Palabras como participación, mejoramiento de las condiciones de vida, etc., carecen de una significación unívoca dada su vinculación deontológica con las personas que las utilizan (Porzecanski, 1983, 39-40). Semejantes reflexiones las aplica después a la relación existente entre los programas de desarrollo de la comunidad y el sistema socio-político que los soporta.

En otro lugar de la obra que estamos siguiendo nos encontramos con una sugerente conclusión sobre las causas del fracaso de algunos programas de desarrollo: «los cambios tecnológicos que puede aceptar un grupo humano dependen del substractum no-material al igual que de su propio condicionamiento material: de sus va- 
lores, creencias, modos de vida, prejuicios, costumbres, etc.». Con ésto se pone de manifiesto lo que Boas denominó teoría del filtro, que explica cómo las pautas de conducta de una comunidad actúa de filtro frente a todo tipo de cambios culturales (citado en Porzecanski, 49).

No menos interesante es la idea de comunidad real, concepto que emplea para la auténtica distinción de las comunidades humanas en base a criterios no exclusivamente geográficos: «comunidad real es el ámbito subcultural dentro del cual es factible lograr una repercusión participativa si se aplica el procedimiento de Organización y Desarrollo de Comunidad» (p. 53).

Pasamos ahora a revisar la obra de otro autor del continente americano, Enrique Oteiza, experto en temas de desarrollo y actualmente colaborador directo de la UNESCO.

La obra de Oteiza (1983) es el resultado de la recopilación de diversos estudios críticos en busca de alternativas a los modelos de desarrollo más ortodoxos, de clara orientación economicista o como él mismo dice de capitalismo dependiente.

Hay una idea que subyace en toda la obra y que constituye el eje de todo el estudio: no confundir el desarrollo con el crecimiento económico y la modernización que sólo beneficia a minorías privilegiadas dejando al resto de la población en situación de pobreza denigrante y privada de las necesidades básicas (Oteiza, 1983, p. 310).

Por otro lado, insiste en la distinción entre desarrollo de la comunidad y los métodos totalitarios para alcanzar el crecimiento ya que suelen ser insensibles a la necesidad de los pueblos de implicarse en el proceso de forma creativa.

Señala la nueva tendencia hacia procesos de desarrollo que aspiran a nuevas formas de equidad social y humanismo, lo que supone la satisfacción de necesidades materiales y la posibilidad de aplicar la energía creativa de la comunidad. Tales aspiraciones suponé la eliminación de las barreras estructurales en las relaciones sociales nacionales e internacionales que impidan la consecución de los nuevos objetivos (Oteiza, 1983, 311).

Más adelante define el desarrollo en estos términos:

«proceso de cambio social basado en un esfuerzo creativo y participativo de los propios pueblos, y en la movilización de los recursos a su disposición, con el fin primero de eliminar la pobreza y la marginalidad, la superación de la explotación y la dominación sociopolítica interna o externa y el continuo despliegue de la personalidad humana a través de su expresión propia» (Oteiza, 1983, 311).

Tampoco podemos olvidar la obra de Natalio Kisnerman llena de conceptualizaciones precisas que nos ayudan a conseguir una más exacta comprensión de los procesos de promoción comunitaria.

Precisamente, una primera aportación radica en el término que acabamos de senalar, el de promoción, que da nombre a la acción ejercida por los trabajadores sociales en y con la población. Para este teórico, promoción equivale al estímulo que recibe la población para que ésta se convierta en auténtica vecindad que toma conciencia de su problemática colectiva, de sus capacidades como colectividad y de sus potencialidades para la resolución de esos problemas (Kisnerman, 1986, 68).

Más adelante se hace más explícito aún al definir la promoción comunitaria. 
«La promoción comunitaria es un proceso de capacitación democrática, en la cual los hombres analizan sus problemas, buscan soluciones e intervienen en las decisiones que les afectan, lo que desarrolla la conciencia de sus cualidades, potencialidades y posibilidades y les permite asumir la responsabilidad de su propio desarrollo individual y colectivo» (Kisnerman, o. c., 69).

Plantea con mucha originalidad el tema de planificación de las acciones de promoción comunitaria como construcción progresiva a largo plazo. Se ha de comenzar con proyectos concretos con capacidad de generar a su vez nuevos proyectos que en conjunto abarquen la totalidad de la problemática. De esta manera, dice Kisnerman, «resulta una planificación que no se impone sino que se va articulando a través de aquellos proyectos que satisfacen necesidades y expectativas concretas de la población» (o. c., 68-69).

También aprecia una matización diferencial entre comunidad organizada y comunidad promovida. Está organizada cuando la población se encuentra capacitada para acometer la solución de los problemas. Cuando además asume la decisión de resolverlos, se dice que está promovida (o. c., 69).

Finalmente, señalamos los requisitos que este autor destaca en la proción comunitaria:
a) Apoyo oficial.
b) Asesoría técnica.
c) Disponibilidad de recursos.
d) Una organización que integre los diferentes proyectos y recursos.
e) Participación (Kisnerman, 1986, 70).

\section{Teóricos del área Europea}

Un autor próximo a nosotros es Marco Marchioni. Sus casi treinta años de dedicación al trabajo social, fundamentalmente en su vertiente práctica, y su presencia en programas que se han desarrollado en nuestro país, justifican sobradamente su inclusión en este capítulo del trabajo.

Lo primero que observamos en su obra $(1987,51)$, por lo que respecta a la conceptualización, es su dedisión de dar un nuevo nombre a la acción social que llamamos desarrollo comunitario. Propone el boloñés una nueva expresión: planificación social y organización de la comunidad. Las razones que esgrime son de naturaleza contextual, teleológica y funcional. Según él, la expresión organización de la comunidad es más adecuada a las condiciones y situaciones actuales, distintas a las de las primeras experiencias. Sin embargo, parece ser que los contextos del desarrollo comunitario y de la organización de la comunidad no se identifican, por lo que ambos conceptos se pueden entender como complementarios (Marchioni, 1987, 52). Su maestra, Angela Zucconi lo precisa al ampliarnos que el contexto al que alude el desarrollo comunitario es una zona rural o deprimida carente de todo tipo de estructura, por el contrario, el contexto al que se aplica la organización de la comunidad es de tipo fundamentalmente urbano donde abundan las estructuras (cf. Marchioni, 1987, 52).

Seguidamente vamos a señalar los principios básicos que según el italiano deben presidir toda acción social comunitaria: 
a) Nunca se podrá hacer un verdadero cambio si no es a través de la plena participación de las personas interesadas.

b) Autodeterminación de los individuos y de las colectividades para elegir su propio camino de desarrollo.

c) El ritmo de desarrollo no debe ser impuesto desde el exterior.

d) La acción social deberá favorecer la maduración colectiva a través de la toma de conciencia de los problemas y la participación de la comunidad en el proceso de mejoramiento de la situación (Marchioni, 1987, 55-58).

En otro lugar de su obra, cuando hace la descripción de un proyecto concreto de desarrollo (municipio de Lebrija), también encontramos interesantes matices teóricos: «el desarrollo no puede ser sectorial y parcial, sino el resultado del avance que los hombres y las mujeres realizan en distintos campos y realidades muy relacionados entre ellos» $(1987,133)$, y más adelante: «el Proyecto promoverá el desarrollo y crecimiento económico al mismo tiempo que el progreso social y cultural de la comunidad y de todos sus miembros».

Finalmente, queremos recoger con especial interés para nosotros una última sugerencia de la obra de este autor por lo que se refiere a este ámbito de lo teóricoconceptual. Se trata de las múltiples alusiones que hace sobre el paralelismo entre procesos de desarrollo y proceso educativo.

En Rudolf Rezsohazy encontramos un firme pilar teórico, práctico y docente en materia de Desarrollo Comunitario. Este autor, profesor de la universidad de Lovaina en la temática que nos ocupa, acumula una sólida experiencia de más de 25 años de dedicación a la investigación y a la puesta en práctica de programas de desarrollo en casi todo el mundo.

De su obra más reciente (Rezsohazy, 1988), interesantísima desde el punto de vista metodológico, entresacamos algunos aspectos teóricos, ciertas nociones básicas que tienen la intención de clarificar conceptualmente la originalidad de los procesos de desarrollo comunitario.

Después de situar históricamente este movimiento, nos ofrece una definición de Desarrollo Comunitario teniendo en cuenta los treinta años transcurridos desde los primeros enfoques de estos procesos:

«El Desarrollo Comunitario es una acción coordinada y sistemática que, en respuesta a las necesidades o a la demanda social, trata de organizar el progreso global de una comunidad territorial bien delimitada o de una población-objetivo, con la participación de los interesados» (Rezsohazy, 1988, 18).

Se extiende después el belga en examinar el alcance de esta definición.

\section{Acción coordinada y sistemática:}

- Persigue unos objetivos precisos.

- Prevé medios e instrumentos para alcanzarlos.

- Planificación precedida de investigación y seguida de evaluación.

- Planificación para alcanzar un cambio deliberado, voluntarista. 


\section{Las necesidades y la demanda social}

- Una de las primeras tareas de toda comunidad que aspira a su desarrollo consiste en conocer sus problemas y formular sus necesidades.

- En la práctica esta tarea no resulta fácil: expresión de deseos no auténticos, mediatizados por la presión social o propaganda; incapacidad de la población para expresar necesidades latentes; ignorancia de otras necesidades esenciales para un observador externo; contexto propicio a la manipulación externa; etc.

- Organización jerárquica de las necesidades.

- Presencia de diálogo con otras esferas: locales, provinciales, estatales, etc.

- Ciertas necesidades colectivas no son perceptibles para la base (Rezsohazy, 1988, 19-21).

\section{El progreso global}

- En el avance de un conjunto social todo depende de todo.

- El desarrollo comunitario debe ser una gestión global e integrada.

- Están llamadas a desplegarse todas las facultades del hombre y mejorar todos los campos de la sociedad.

- Crea para los miembros de la comunidad oportunidades y nuevos recursos materiales, intelectuales y espirituales.

- En definitiva es progreso en el arte de vivir.

\section{La población}

- Entidad geográficamente localizable, que forma una unidad de vida y que comparte equipamientos, servicios, relaciones, etc.

- No considerada como un conglomerado o masa sino en sus interrelaciones vivas, en sus intereses y valores comunes.

- Se presenta como una unidad de acción natural.

\section{La participación}

- Es lo que distingue al desarrollo comunitario de otros métodos.

- Supone el acceso de los hombres a las decisiones que les afectan.

- Implica: concienciación de los propios problemas: reconocimiento del cambio social desde el propio fondo cultural; búsqueda común de soluciones; creación de dinamismo que puede suscitar nuevas iniciativas (Rezsohazy, 1988, 22-24).

Centramos ahora nuestra atención en Albert Meister, sociólogo mundialmente conocido en el ámbito del desarrollo y la autogestión tanto por sus abundantes aportaciones teóricas como por su labor práctica en distintas experiencias de Africa, América Latina, Israel, Yugoslavia, etc.

La producción escrita de Meister es abundantísima pero nosotros vamos a seleccionar lo más significativo de su pensamiento en una obra-homenaje que sus alumnos y seguidores le han dedicado por su reciente fallecimiento (Varios, 1988). 
Todo el análisis de A. Meister concerniente al desarrollo comunitario y a la animación rural descansa sobre un principio básico que podría resumirse así: adoptar una posición crítica en un universo explotado (Le Boterf, 1988, 63). Esta postura crítica se deja ver en las observaciones e hipótesis de trabajo que del sociólogo galo selecciona Le Boterf:

a) Las estructuras tradicionales constituyen un impedimento al desarrollo económico; 'éste exige una cierta ruptura con ellas. Se refiere a las estructuras conservadoras que no tienen ninguna voluntad de cambio.

b) Consecuentemente, toda acción de desarrollo que se asiente sobre estas estructuras no puede generar procesos duraderos de cambio.

c) La formación, presente en el centro de los proyectos de animación y desarrollo, por sí misma no puede generar acción. Es un gran recurso cuando trata de adaptarse al cambio pero es insuficiente para provocarlo e impulsarlo.

d) Las administraciones, como las que ahora tenemos, constituyen un obstáculo para el desarrollo.

e) Las acciones de desarrollo exigen análisis microeconómicos o culturales precisos donde se tome en cuenta la especificidad de cada situación.

f) Las operaciones de desarrollo llamadas participativas están de hecho prisioneras de una programación demasiado rígida.

g) El desarrollo, guste o no, supone braceo, selección de los más dinámicos, espíritu de empresa, iniciativas individuales, estímulo del esfuerzo individual. Estos valores son poco apreciados por el desarrollo comunitario y la animación rural (Le Boterf, 1988, 65). Quizá haya que añadir que A. Meister se refiere al desarrollo de los años sesenta-setenta y que su labor, junto a la de otros tantos, haya servido para llegar a un modelo de desarrollo, el de la década de los ochenta, más acorde con lo que él mismo exigía.

Otro autor del área europea que nos hace serias aportaciones en nuestro tema de investigación es Pierre Furter. Actualmente, el profesor Furter imparte sus enseñanzas en la universidad de Ginebra, donde continúa sus investigaciones sobre desarrollo regional y otros temas próximos como la educación permanente y la educación de adultos en relación con el desarrollo de los pueblos, las disparidades regionales en el desarrollo de la educación, etc. También cuenta con amplia experiencia práctica en el campo de la acción socioeducativa en países de América Latina.

Aunque después volvamos a su producción científica, ahora sólo nos detendremos en los aspectos más significativos en cuanto a las bases conceptuales del Desarrollo Comunitario. En relación a ésto, encontramos en una de sus obras más conocidas (Furter, 1983) una oportuna diferenciación entre los conceptos de Desarrollo Comunitario y Animación Rural: «ambas intervenciones se parecen hasta el punto de poder creer que se trata del mismo tipo de acción» (Furter, 1983, 190).

Furter nos ofrece lo que entiende por Desarrollo Comunitario al tratar de distinguir las intervenciones aludidas, aunque basándose en opiniones de otros autores como Goussault y Meister: 
- El Desarrollo Comunitario, ante todo es una acción socio-educativa.

- Tuvo su contexto histórico en la independencia del régimen colonial anglosajón al tener que hacer frente las comunidades a las necesidades más inmediatas sin el amparo de la metrópoli.

- Las necesidades son definidas por la propia población sin atenerse a otros contextos, regionales o nacionales.

- No está organizado a nivel de red nacional ni coordinado directamente a la acción de otros servicios técnicos.

- Responde directamente a los problemas que las colectividades están llamadas a resolver en función de su propio ritmo de desarrollo (Furter, 1983, 190).

Respecto a la conceptualización de la Animación Rural, señala interesantes matices que los veremos más adelante en otro apartado más indicado.

Antes de entrar en la aportación de los autores españoles queremos recoger algunas puntualizaciones útiles del italiano Giacomo Viccaro, impulsor en toda el área mediterránea de la metodología denominada Investigación Participativa, forma diferente de educación de adultos propuesta por el Consejo Internacional de Educación de Adultos (ICEA) y bastante emparentada, metodológicamente, con el Desarrollo Comunitario.

Hace el italiano interesantes distinciones entre desarrollo económico, desarrollo y progreso (Viccaro, 1986, 152-154).

Se suele identificar con mucha frecuencia, nos dice, el desarrollo con el desarrollo de los hechos exclusivamente económicos, es decir, con el desarrollo económico, lo que evidencia la importancia de las variables económicas en la vida del individuo y de las comunidades, sobre todo en épocas de crisis. No obstante, admitiendo la importancia del desarrollo económico en el desarrollo de la comunidad, éste último va más lejos de aquel al contemplar factores de carácter extraeconómico como son la población, su composición, el orden político-social, el grado de instrucción, etc. (Viccaro, 1986, 152).

Asume las palabras del secretario general de la UNESCO, A. Mathar M'Bow, pronunciadas en la Conferencia Internacional sobre educación de Adultos (París, octubre, 1982): «El desarrollo se basa en el hombre y comprende todas las capas de la sociedad, es decir, la sociedad entera. En resumen, el desarrollo ha de incitar a la comunidad a ofrecer a todos sus miembros la posibilidad de participar».

Para Pasolini (Viccaro, 1986, 153), el término progreso es más adecuado para expresar la promoción humana a nivel ideal, social, política, etc. Propone reservar el término desarrollo, dada su equivocidad, para los aspectos estrictamente económicos. El desarrollo ha de ir acompañado del progreso, de lo contrario sería una constante fuente de disociación.

El progreso, según Viccaro, es:

«el único desarrollo merecedor de ser continuado, social, política y humanamente; es decir, un desarrollo que refuerce el desarrollo material al mismo tiempo que todos los componentes positivos, de carácter no económico, comprometiendo al hombre y a todos los hombres en la vida individual y colectiva en las relaciones sociales y, en resumen, en todas las modalidades donde se exprese la presencia humana en el ámbito de la vida» (Viccaro, 1986, 153). 


\section{Teóricos españoles}

Dentro del panorama español, comenzaremos por las aportaciones del profesor J. M. ㅁ Quintana Cabanas, autoridad indiscutible en el campo de la Pedagogía Social dentro y fuera de nuestro país. Su obra teórica y docente viene impulsando, primero desde la Universidad Autónoma de Barcelona y ahora desde la U.N.E.D., la investigación del Desarrollo Comunitario como una nueva manera de entender la educación bajo una perspectiva liberadora.

Queremos destacar el enfoque pedagógico que el profesor Quintana da al Desarrollo Comunitario ya que en definitiva esta es la faceta que más queremos evidenciar a lo largo de nuestro trabajo: «El proceso de liberación de los grupos humanos oprimidos ha de pasar necesariamente por un proceso de educación» (Quintana Cabanas, 1988, 93). En otro lugar leemos: «El llamado Desarrollo Comunitario o de la Comunidad constituye una prolongación del desarrollo individual ...»; «... la idea central ... representa la superación de una realidad humana actual, llevándola a niveles superiores de perfeccionamiento y de calidad de vida» (prólogo a Rezsohazy, 1988, 9).

Nos apunta el pedagogo catalán algo que debemos tener en cuenta al hablar de Desarrollo Comunitario, su doble interpretación, como fin o como medio. Desde él primer punto de vista sería la utópica aspiración deseable como punto terminal de todo colectivo humano deprimido; desde el segundo, se entiende como instrumento técnico, como intervención que goza de sus propios métodos y técnicas de acción (prol. a Rezsohazy, 1988, 9).

También nos señala una realidad que venimos constatando, el Desarrollo Comunitario suele compartir espacios de intervención comunes a otras técnicas: educación de adultos, participación comunitaria, desarrollo regional, etc. (Quintana, 1984,$505 ; 1986$ b 9). Pero no sólo nos plantea el problema sino que nos ayuda a resolverlo con finas aportaciones conceptuales:

«... Hemos ido constatando una idea de la educación de adultos, entendida como: a) concienciación de los problemas sufridos por el individuo; b) análisis de sus causas sociales, y c) intervención activa en el entorno para remediar esos problemas. Dicha idea, que constituye el concepto actual y universal de la educación de adultos, da lugar a un movimiento llamado desarrollo comunitario, y se plasma en un método llamado investigación participativa» (Quintana, 1984, 505).

«La investigación participativa es una metodología de la educación de adultos para promover el Desarrollo Comunitario, conseguir la participación de los adultos en la resolución de sus problemas sociales...» (Quintana, 1986b, 10).

El Departamento de Pedagogía Sistemática de la Universidad de Santiago de Compostela ha sido uno de los primeros de nuestro país que ha impulsado los estudios sobre las distintas intervenciones socioeducativas. Un profesor de este Departamento, Agustín Requejo Osorio, ha elaborado algunos trabajos sobre Desarrollo Comunitario dignos de tenerse en cuenta en este apartado de las aportaciones de los teóricos.

En la introducción de uno de estos trabajos (Requejo, 1988b), presenta el Desarrollo Comunitario como una nueva coordenada de la educación actual como consecuencia de la tendencia hacia los sistemas escolares más abiertos que se origina 
en la década de los sesenta. Nos habla de dimensiones nuevas de este proceso educativo más flexible: participativo, descentralizado, integral, incluyendo las demandas sociales, contribuyendo a todas las formas de desarrollo de la personalidad del hombre.

La educación, bajo las nuevas perspectivas, ha de hacerse eco de las necesidades cotidianas y concretas de la población. Se abren nuevos horizontes. Se proyecta hacia la dimensión de la comunidad y su desarrollo (Requejo, 1988b, 2).

Citando a Fletcher, nos señala un nuevo concepto en este amplio panorama, educación comunitaria o participación de las comunidades en la educación una vez que éstas se interesan por conservar el patrimonio cultural que las identifica y consolida. Pero que también contribuye al desarrollo de las mismas desde el momento en que se dedica a promover actividades encaminadas al bienestar de todos sus miembros $(1988 b, 3)$. Más adelante insiste: «... La educación comunitaria está por tanto en la dirección y en el contexto del Desarrollo Comunitario. Es la propia comunidad el agente, el punto de arranque, convergencia y proyección para alcanzar sus propios proyectos» (p. 4).

También resulta de interés la precisión que hace a continuación basándose en una cita de Summers en la que ubica los aspectos económicos del desarrollo en el concepto desarrollo en la comunidad, perspectiva que insiste en el crecimiento económico que conlleva todo desarrollo; concepto que difiere del desarrollo de la comunidad, que destaca el reforzamiento del espacio comunitario a través de una serie de canales de comunicación y cooperación entre los grupos locales (Requejo, 1988b, 4).

Nos propone el profesor Requejo, en otro de sus trabajos, otra conexión entre educación y Desarrollo Comunitario. En esta ocasión vinculándolo a la educación permanente según las recomendaciones de la IV Conferencia Internacional sobre Educación de Adultos (París, 1985), que invitan a incluir el desarrollo local en la planificación y política educativa destinada a los adultos (Requejo, 1988a, 2).

Como sólo nos interesa, por ahora, lo relacionado con el ámbito conceptual, recogemos finalmente otra aportación que viene a sumarse a nuestra tesis:

«... El auténtico desarrollo es, por tanto, fundamentalmente desarrollo humano equilibrado e integral, que abarca tanto el sector económico como el político, social, educativo y administrativo y se inscribe en un espacio democrático. Comprende, no sólo la satisfacción de las necesidades básicas (alimentación, salud, vivienda...), sino el aumento de los niveles de vida (trabajo, ingresos, educación, cultura...) y las condiciones de emancipación y libertad (económico-social)» (Requejo, 1988a, 5).

No podemos olvidar, dentro de la Universidad de Santiago, las aportaciones de otros investigadores como José Antonio Caride Gómez (1986a, 1986b), Mª Dolores, F. Candedo (1986), etc. cuyos trabajos se orientan hacia el enfoque de los procesos de animación y desarrollo como nuevas formas de educación. 


\section{BIBLIOGRAFÍA}

ANDER-EGG, E. (1986): Metodología y práctica del Desarrollo de la comunidad. Ateneo, México.

CANDEDO, Ma D. (1986): «Bases para un proyecto de intervención socioeducativa a nivel local». En QUINTANA, J. M.- (1986): Fundamentos de Animación Sociocultural. Narcea, Madrid.

CARIDE, J. A. (1986): «La Animación sociocultural en zonas deprimidas». En Referencias. Ministerio de Cultura. Madrid.

- (1986): «Educación y Animación Sociocultural». En QUINTANA J. Ma (1986): Fundamentos de Animación Sociocultural. Narcea, Madrid.

FURTER, P. (1983): Les espaces de la formation. Presses Polytechniques Romandes. Laussanne.

- (1986): Un Siecle d'Interventions Socioculturelles en Espagne (1876-1986). Faculté de Psychologie et des Sciences de 1'Education. Université de Genève.

KISNERMAN, N. (1986): Comunidad. Humanitas, Buenos Aires.

LE BOTERF, G. (1988): «La participation et le developpement: l'analyse d'A. Meister et les tendences Actualles». En VARIOS: L'autogestion dissait-on! Presses Universitaires de France, Paris. Cahiers de L'I.U. D.E. n⿳ำ 18. Genève.

MARCHIONI, M. (1987): Planificación social y organización de la comunidad. Popular, Madrid.

OTEIZA, E. (1983): Autoafirmación colectiva: una estrategia alternativa de desarrollo. F.C.E. México.

PORZECANSKI, T. (1983): Desarrollo de comunidades y subculturas. Humanitas, Buenos Aires.

QuinTANA, J. Ma (1984): Pedagogía Social. Dykinson, Madrid.

- (1986): Fundamentos de Animación Sociocultural. Narcea, Madrid.

- (1988): «La educación permanente en Europa». En VARIOS: Una educación para el desarrollo: la Animación Sociocultural. Fundación Banco Exterior de España, Madrid.

- (1988): Teoría de la educación. Concepción antinómica de la educación. Dykinson, Madrid.

REQUEJO, A. (1989): «La intervención pedagógico-social y el desarrollo comunitario». En Pedagogía Social, no 4 (169-179). Universidad de Murcia.

- (1988): «Desarrollo Comunitario y Educación» (documento policopiado). Dpto. de Teoría e Historia de la Educación. Universidad de Santiago de Compostela.

REZSOHAZY, R. (1988): El desarrollo comunitario. Narcea, Madrid.

RoDríGUEZ, C. (1970): Análisis Conceptual del Desarrollo Comunitario. ECRO, Buenos Aires.

VICCARO, G. (1986): «El rol de los educadores de adultos según la experiencia de la región de Toscana». En Educar, no 9. Universidad Autónoma, Barcelona.

- (1983): Educazione e decentramento. Ligouri, Nápoles.

VARIOS (1988): L'autogestion dissait-on! Presses Universitaires de France, París. Cahiers de L'I.U.D.E. nº 18 . Genève.

WARE, C. (1954): Organización de la comunidad para el Bienestar Social. Unión Panamericana. Washington. 